\title{
PRESUPOSICIONES Y COMPLETIVAS DE SUJETO (CON PREDICADOS DE AFECCIÓN PSÍQUICA)
}

\author{
Carmen Cabeza \\ (Universidad de Vigo)
}

\section{RESUMEN}

Presupposition has been described, in terms of logic, as a relation between two utterances. The classical example is: The present king of France is bald, which presupposes: There exists at present a king of France. Presuppositions are related to some expressions called presuppositional actualizers. Factive predicates (to lament, to be sorry that, to worry, etc.), which have the property of introducing subordinate clauses with a meaning which is presupposed to be true, are presuppositional actualizers. They are called factives because the clause depending on them can be introduced by the phrase the fact. Other properties which have been described for these predicates are the possibility of the embedded clause being preposed and (in Spanish) the presence of the subjunctive mood in this subordinate.

It is important to stress the importance that the concept of presupposition has for the analysis of these sentences from a pragmatic point of view. The speaker takes a part of what he/she says as something already known, as a background on which another item of information is salient. This item of information is not presupposed any more, it is stated as true.

In this article we examine a subgroup inside the factive predicates in Spanish: those of psychic affection (preocupar -to worry-, extrañar -to amaze-, sorprender -to surprise-, divertir -to amuse-, etc.). All these can be accounted for the scheme SUJ-PRED-CIND (Subject, Predicate, Indirect Object) in the active voice.

We argue that the predicates of affection are prototipically factives. In contrast with those which have a subordinate in other functional roles the three formal properties mentioned before are present here.

This leads us to advance the hypothesis that there exists a relation between the capacity of these verbs of activating presuppositions and the fact that the subordinate clause is made the grammatical Subject.

\section{Introducción}

Las estructuras sintácticas son moldes formales a través de los cuales las lenguas organizan o disponen determinados contenidos. Si alguien en nuestra 
presencia pronuncia un enunciado como Elisa cultiva fresas en su huerta nos comunica que una persona llamada Elisa (que podemos conocer o no, eso es lo de menos) ejecuta una acción sobre un objeto en un determinado lugar. Podemos decir que la secuencia Elisa cultiva fresas en su huerta representa un proceso. Pero además se dicen otras cosas a través de ella. Por ejemplo, que existe alguien identificable a través de un nombre propio y que tiene una huerta. La presencia del nombre propio Elisa presupone la existencia de la persona por él designada y el posesivo su presupone la existencia de una huerta que es de su propiedad.

Las presuposiciones son objeto de estudio por parte de los lógicos y también de los lingüistas. Entre las disciplinas lingüísticas es la pragmática la que se ocupa más directamente de ellas. Aquí vamos a ver cómo determinado tipo de presuposiciones (las que aparecen asociadas a los llamados predicados factivos) pueden tener interés para el estudio de ciertas construcciones en que se insertan esos predicados, en concreto, de las cláusulas con un sujeto y un complemento indirecto (esquema SUJ-PRED-CIND) en que el sujeto está desempeñado por una completiva.

\section{La presuposición}

La presuposición es la relación lógica entre dos enunciados, E1 y E2, tal que si E1 es verdadero, E2 es verdadero, y si E1 es falso, E2 es igualmente verdadero, es decir, E2 tiene que ser verdadero para que E1 tenga valor de verdad. El ejemplo clásico de presuposición es El actual rey de Francia es calvo.

E1: El actual rey de Francia es calvo.

E2: Existe actualmente un rey de Francia.

En unas circunstancias históricas en las que E1 se ajuste a la realidad, E2 es necesariamente verdadero. En el supuesto de que haya rey en Francia, y que éste no sea calvo (E1 falso), E2 sigue siendo verdadero. Ahora bien, si E2 es falso (no existe actualmente un rey de Francia) entonces E1 no es ni verdadero ni falso.

En la lógica se reconocen tres tipos de presuposiciones (Deaño 1980: 307309):

1) Las que se derivan de la utilización de una frase nominal con referente específico (es decir, que se refiera a un objeto o individuo fácilmente identificable). Ejemplo: El actual rey de Francia es calvo.

2) Las que tienen lugar en los enunciados universales. Ejemplo: Todos los que intentan hacer compatible el cristianismo con el marxismo son en el fondo cristianos (EI) presupone Existen personas que intentan hacer compatible el cristianismo con el marxismo (E2).

3) Las que aparecen en las proposiciones subordinadas a predicados como darse cuenta, lamentar, olvidar, etc. Ejemplo: El general se dio cuenta de que 
sus tropas le habian abandonado (E1) presupone Sus tropas le habian abandonado (E2).

En el análisis lingüístico se utiliza la negación como prueba de la presencia de presuposiciones. Para probar si el enunciado El general se dio cuenta de que sus tropas le habian abandonado (E1) presupone en efecto Sus tropas le habian abandonado (E2) se observa qué ocurre si negamos E1: El general no se dio cuenta de que sus tropas le habian abandonado. A pesar de la negación la presuposición se mantiene.

Una característica importante de las presuposiciones es que aparecen vinculadas a determinados elementos de la estructura lingüística, que Levinson (1983) llama accionadores presuposicionales. Propone la siguiente clasificación':

\section{Descripciones definidas.}

Las descripciones definidas son frases nominales en singular con determinante definido. A diferencia de los nombres propios (que aluden siempre a un individuo o un objeto concretos), las descripciones definidas pueden no tener referencia. Son descripciones definidas las frases en negrita en los siguientes ejemplos: Miguel de Cervantes es el autor del Quijote, El rey de Francia es calvo o John vio al hombre con dos cabezas. Según Strawson (1950), los enunciados con descripciones definidas implican en cierto sentido (esto es, presuponen) que existe el referente que designan. Así, por ejemplo, John vio/ no vio al hombre con dos cabezas presupone existe un hombre con dos cabezas.

2. Verbos factivos (lamentar, sentir, ser consciente, darse cuenta, saber, etc. $\left.{ }^{2}\right)$ :

John se dio cuenta / no se dio cuenta de que estaba en deuda presupone John estaba en deuda.

3. Verbos implicativos (conseguir, olvidar, evitar):

John consiguió / no consiguió abrir la puerta presupone John intentó abrir la puerta.

4. Verbos de cambio de estado (dejar de, empezar a):

John dejó / no dejó de pegar a su mujer presupone John pegaba a su mujer.

5. Expresiones con valor iterativo (ya no, de nuevo, otra vez, repetir, volver), por ejemplo:

El platillo volante volvió / no volvió a venir presupone El platillo volante vino antes; Ya no se pueden encontrar pirulís presupone Hubo un tiempo en que se podian encontrar pirulís.

1 Los ejemplos proceden de Levinson (1983).

2 Son algunos de los verbos factivos que Levinson menciona, citando a Kiparsky y Kiparsky (1971). Ser consciente, darse cuenta o saber estarían entre los predicados factivo-asertivos (semifactivos) en la clasificación de Hooper (1975). Aquí consideraremos como prototípicamente factivos a los predicados de afección psíquica (divertir, entretener, molestar, sorprender, etc.)

Levinson pone en duda el carácter presuposicional de estas implicaciones, debido a que no se pueden atribuir al hablante sino al sujeto del juicio. 
6. Verbos de juicio (acusar, criticar) ${ }^{3}$ :

Agata acusó a John de plagio presupone (Agata cree que) el plagio es malo. 7. Cláusulas temporales (introducidas por antes, mientras, desde que, después, etc.), como:

Mientras Chomsky estaba revolucionando la lingüística, el resto de la ciencia social estaba / no estaba dormido presupone Chonsky estaba revolucionando la lingüistica.

8. Construcciones escindidas (o construcciones ecuacionales ${ }^{4}$ ). Son recursos sintácticos de focalización de un elemento de la cláusula:

Fue / no fue Henry quien besó a Rosie (construcción escindida) presupone Alguien besó a Rosie (no escindida).

9. Escisiones implicitas con constituyentes enfatizados. Un constituyente de una cláusula se puede enfatizar (y entonces se consigue el mismo efecto de focalización que se obtiene con las construcciones escindidas) mediante el uso de un determinado patrón entonativo:

John compitió / no compitió EN LAS OLIMPIADAS presupone John compitió en algún lugar.

10. Comparaciones y contrastes. También las estructuras comparativas, así como ciertas expresiones de contraste, dan lugar a presuposiciones:

Jimmy es / no es tan impredeciblemente torpe como Billy presupone Billy es impredeciblemente torpe; Adolph llamó a Marianne valquiria, y ella lo piropeó a su vez / a cambio / también presupone Llamar a alguien (o al menos a Marianne) valquiria es piropearla.

11. Cláusulas de relativo no restrictivas (o explicativas):

Los proto-harrapanos, que florecieron en los años 2800-2650, fueron / no fueron grandes constructores de templos presupone Los proto-harrapanos florecieron en los años 2800-2650.

12. Condicionales contrafactuales (o irreales):

Si Aníbal hubiera tenido solamente doce elefantes más, hoy en día existirían/no existirian las lenguas románicas presupone Aníbal no tenía doce elefantes más.

13. Preguntas.

En este punto distingue Levinson entre el efecto presuposicional de las interrogaciones totales (cuya respuesta es sí o no) y el de las preguntas parciales (con pronombres interrogativos: quién, dónde, cómo, etc.). Llama presuposiciones vacuas a las primeras y no vacuas a las segundas 5 .

Ejemplo del primer tipo: ¿Hay catedrático de Lingüística en el MIT? presupone $O$ bien hay catedrático de Lingüistica en el MIT o bien no lo hay.

\footnotetext{
4 Vid. Martínez (1984).
}

5 Advierte Levinson que las presuposiciones que se derivan de las interrogativas parciales no son invariables bajo los efectos de la negación (1983: 176). 
Ejemplo del segundo tipo: ¿Quién es el catedrático de Lingüística en el MIT? presupone Alguien es el catedrático de Lingüística en el MIT.

Una tercera propiedad de las presuposiciones es que pueden cancelarse por la acción del contexto. Así, hemos visto que los conectores temporales son accionadores presuposicionales:

Sue lloró antes de terminar su tesis presupone Sue terminó su tesis

Sin embargo la presuposición queda sin efecto en

Sue murió antes de terminar su tesis (ejemplos de Levinson).

\section{Los verbos factivos}

Entre esta variedad de accionadores presuposicionales nos ocuparemos en particular de los verbos factivos. El interés por este tipo de predicados surge en el seno de la Gramática Generativa (teoría estándar extendida) a raíz de la publicación de un artículo de Paul y Carol Kiparsky titulado Fact (= hecho) ${ }^{6}$. Estos autores concibieron la presuposición como un rasgo de carácter semántico (presente en la estructura profunda) que permitía explicar ciertos fenómenos que se daban en la estructura superficial. De esta manera conseguían vincular la sintaxis con la semántica, lo cual constituía una de las aspiraciones dentro del marco de la corriente generativa. Las completivas dependientes de predicados factivos tendrían, según Kiparsky y Kiparsky, la palabra fact en su estructura profunda ${ }^{7}$. Oraciones como I regret the fact that John is ill (= «lamento el hecho de que John esté enfermo») tienen una estructura superficial similar a su estructura profunda. En cambio, para explicar la oración I regret that John is ill (= «lamento que John esté enfermo») es preciso aplicar una transformación de borrado sobre la palabra fact.

Demonte (1977), también desde la GT, reconoce las siguientes propiedades sintácticas en las oraciones con predicados factivos (ejemplos de la autora):

1) La posibilidad de introducir la subordinada con la frase el hecho o el artículo el:

Es triste el que / el hecho de que el loro tenga gripe

2) La movilidad dentro de la cláusula. Sólo los predicados factivos permiten que la completiva ocupe la posición inicial:

Que a mi madre le duela la cabeza me preocupa

3) La imposibilidad de los fenómenos de raising (= «ascenso»), que sí tienen lugar con otros predicados:

( Kiparsky y Kiparsky (1971).

7 Kiparsky y Kiparsky describen las estructuras del inglés. Violeta Demonte, en su estudio sobre la subordinación sustantiva (Demonte 1977) habla de un tipo particular de predicados del español en cuya estructura profunda aparece siempre la frase el hecho.

\& Esta propiedad es consecuencia del carácter de islas sintácticas que tienen las subordinadas dependientes de predicados factivos (Kiparsky y Kiparsky 1971: $\$ 4$; Demonte 1977: $\$ 5.2 .1$ ). 
Imagino que él viaja hacia Bruselas $\rightarrow$ Lo imagino viajando hacia Bruselas (raising con verbo no factivo)

Lamenté que el médico no viniera $\rightarrow$ *Lo lamenté no venir (raising imposible con predicado factivo).

Para Demonte son predicados factivos en español: lamentar, ser sorprendente, preocupar, descubrir, advertir, ser raro, ser interesante, ser trágico.

\section{Clasificaciones semánticas de predicados. La presuposición y el modo}

Otra propiedad sintáctica que se ha puesto en relación con las presuposiciones vinculadas a los verbos factivos es la sclección de una determinada scrie modal en el verbo de la cláusula subordinada.

La selección del modo en las completivas se ha interpretado con frecuencia como un fenómeno de rección, esto es, como una determinación sintagmática, motivada por algún aspecto de significado del verbo regente. Así, por ejemplo, Fernández Ramírez (1986: 320-321) propone generalizaciones como las siguientes":

a) Rigen indicativo:

1) Los verbos que significan «percepción material o intelectual» ${ }^{10}$. Por ejemplo: ver, oir, sentir, advertir, observar, comprobar, olvidar, pensar, decir, saber, conocer, intuir, prever, considerar, reconocer, ctc.

2) Los verbos que significan «transmisión de información o conocimientos»: decir, declarar, escribir, comunicar, enseñar, contar, exponer, relatar, afirmar, confesar, revelar, responder, avisar, indicar, señalar, etc.

3) Los que expresan «juicios» y «operaciones intelectuales objetivantes» ${ }^{11}$, como deducir, descubrir, averiguar, dar por supuesto, fundamentar, sacar en consecuencia, dejar sentado, describir, etc.

b) Por el contrario, rigen subjuntivo:

1) «Los verbos que indican en sí mismos negación». Por ejemplo: olvidar, ignorar, desmentir, ocultar, etc.

2) Los «verbos voluntativos tanto si con ello se declara o no la voluntad» $»^{12}$ : pedir, pretender, permitir, rogar, suplicar, mandar, ordenar, intentar, tolerar, sufrir, consentir, etc.

3) Los que significan «sentimiento o emoción»: aplaudir, extrañar, temer. significado.

Advierte además que hay casos en que un cambio de modo repercute en un cambio de

10 Entrecomillado del autor.

11 Entrecomillado del autor.

12 Id. 
Bybee y Terrell (1974) proponen una «hipótesis semántica» para explicar la variación modal en la subordinación. Defienden que el modo de la subordinada es un reflejo de la actitud del hablante y también se elige libremente. Como consecuencia de ello, tiene significado por sí mismo. Esta postura pretende evitar la consideración de la rección modal en la completiva como un fenómeno puramente mecánico.

Ofrecen una clasificación de las oraciones en seis tipos, de acuerdo con criterios semánticos y sintácticos. La presuposición, que conciben como una noción de carácter semántico, les proporciona el punto de partida.

Observan que existe una diferencia importante entre presuponer que una proposición es verdadera y afirmar que lo es. En el ejemplo Es verdad que María es guapa se afirma que «María es guapa», pero no se presupone. Si se utiliza la negación como indicio de la presencia de presuposición se verá que esto es así, puesto que se altera el valor de verdad del contenido de la cláusula subordinada:

No es verdad que María es/sea guapa ${ }^{13}$.

No es posible (dicen los autores) que un mismo enunciado se afirme y se presuponga al mismo tiempo. Afirmación y presuposición se excluyen mutuamente, y pueden servir por lo tanto para distinguir tipos semánticos de oraciones, especialmente si se descubre algún tipo de relación entre ambas nociones y el uso de los modos indicativo y subjuntivo en la subordinación. Esto es lo que se proponen a continuación Bybee y Terrell. Sin embargo, no encuentran una relación directa entre una serie modal y la presuposición, ya que algunos verbos factivos se construyen con indicativo y otros con subjuntivo ( $y$, como veremos a continuación, esto les plantea un problema que no son capaces de resolver). La hipótesis a la que llegan es por lo tanto más débil: asocian la aserción con el modo indicativo y la no-aserción con el subjuntivo.

Las oraciones asertivas se construyen, pues, con el modo indicativo. Pueden expresar una afirmación fuerte (con predicados como es seguro o es verdad) o una afirmación débil (por ejemplo, con creer o parecer). También comunican aserciones algunos verbos como decir, leer, contestar, contar. Tienen de particular que especifican el medio a través del cual se transmite la afirmación. Las oraciones que transmiten una afirmación de manera directa se identifican bajo la etiqueta semántica «creencia», mientras que las que especifican el modo bajo el que se comunica la afirmación son denominadas de «informe».

No son aserciones otros dos tipos de enunciados: los de mandato y los de duda. Ambos pueden expresarse a través de una cláusula compleja (más fre-

13. Ejemplos de Bybee y Terrell (1974). 
cuentemente los segundos). El modo de la subordinada es el subjuntivo. Algunos verbos que introducen mandatos son: preferir, aconsejar, permitir, ser necesario, etc. Expresan duda: dudar, ser posible, negar, y también las formas negadas de los predicados de creencia: no parecer, no creer, etc.

En otro apartado de la clasificación se encuentran las oraciones que expresan un «juicio de valor», como, por ejemplo, Es una lástima / bueno / malo / interesante que María no haya podido terminar a tiempo, y también las que transmiten un comentario subjetivo: Me alegro de que María haya podido acabar a tiempo.

Éstas pertenecen al grupo de «comentario», y se construyen habitualmente con subjuntivo. Tienen en común que sus complementos proposicionales se presuponen: si un hablante dice que algo es una lástima (bueno, malo, interesante) o bien que se alegra de algo, es preciso que dé por hecho su valor de verdad, esto es, que lo presuponga.

Pero hay otro tipo de complementos que se presuponen: los que dependen de predicados de «acto mental» (darse cuenta, aprender, tomar en consideración). Estos constituyen un escollo para la aplicación de la hipótesis de Bybee y Terrell ya que, a pesar de que comparten con los de comentario el hecho de ser presuposiciones, llevan el verbo en indicativo, como las afirmaciones. Los autores reconocen que constituyen una excepción:

Los complementos de este pequeño grupo de cláusulas principales son excepciones al principio general de que todos los complementos que se afirman se construyen en indicativo, mientras que los complementos noafirmativos lo hacen en subjuntivo

(Bybee y Terrell 1974: 153)

Advierten, sin embargo, una similitud entre estas oraciones y las asertivas, que justificaría el empleo del indicativo en estos casos. Pero no pueden admitir que son también afirmaciones, puesto que la presuposición y la aserción no pueden coexistir en el mismo enunciado.

La raíz del conflicto está en mantener que la presuposición es un rasgo de carácter semántico. Bybee y Terrell utilizan como criterio para verificar la presencia de presuposiciones la prueba de la negación: si, al negar el predicado regente, se mantiene el valor de verdad del contenido del complemento clausal, hay presuposición. Es precisamente la aplicación de este test, de procedencia lógica, como hemos visto, lo que les lleva a incluir los predicados de acto mental entre los que presuponen la verdad de la proposición contenida en la subordinada, al lado de los de comentario (que suelen ir en subjuntivo), de ahí que no puedan mantener la esperada correlación entre la presencia de aserción o presuposición y la elección de una u otra serie modal.

Para salvar esta dificultad, otros autores han defendido la conveniencia de distinguir dos maneras diferentes de ver la presuposición: desde un punto de 
vista semántico y desde un punto de vista pragmático o discursivo ${ }^{14}$. De acuerdo con la definición lógico-semántica, la presuposición es una relación entre enunciados definible en términos de valores de verdad; según la perspectiva pragmática, las presuposiciones resultan de la «actitud» del hablante.

Sólo una aproximación de tipo pragmático permite oponer la presuposición a la aserción (vid. Kempson 1975: $\$ 3.2$ ): el hablante, o bien afirma algo, o bien lo presupone.

Partiendo de la distinción entre estas dos perspectivas, trata Guitart (1990) de resolver el conflicto ante el que se encontraron Bybee y Terrell. Según Guitart, los predicados de conocimiento y de adquisición de conocimiento, como él los llama (esto es: saber, ser consciente, darse cuenta, notar, descubrir, etc.), cumplen la prueba de la negación, es decir, el contenido de la completiva sigue considerándose verdadero aunque se niegue la principal. Por lo tanto, desde el punto de vista semántico presuponen la verdad de la subordinada. Ahora bien, desde el punto de vista del hablante puede darse el caso de que éste presuponga (dé por consabido) el contenido de la subordinada, o bien puede ocurrir que lo afirme, esto es, que no suponga que el oyente comparte su conocimiento de la situación representada en la subordinada. Observemos el siguiente ejemplo:

Noté que el bar estaba cerrado; no te molestes en ir.

Para decidir si hay presuposición semántica, utilizamos la prueba de la negación, y observamos que sigue siendo cierto que el bar estaba cerrado:

No noté que el bar estaba cerrado

Pero por medio de ese enunciado el hablante informa a su interlocutor de que el bar estaba cerrado y, por lo tanto, puede ser inútil ir (ya que puede seguir cerrado ahora). Desde el punto de vista pragmático, pues, hay afirmación.

Por lo tanto, pueden convivir en un mismo enunciado presuposición semántica y afirmación pragmática.

Otros autores utilizan para los predicados de acto mental la denominación de semi-factivos, lo cual equivale a admitir grados en la factividad ${ }^{15}$. Los predicados de «comentario» (a los que aquí llamaremos de afección psíquica) son más factivos que los de «acto mental» (o de conocimiento).

\section{Los verbos de afección con cláusula de sujeto.}

La sintaxis del español ofrece un tipo de construcción para cuyo análisis resulta adecuada, como veremos, la noción de presuposición ${ }^{16}$.

14 Keenan (1971), Stalnaker (1972), Karttunen (1973), Kempson (1975), Guitart (1990).

15 Por ejemplo, Karttunen (1971), Oh (1974), Hooper (1975). La idea de que algunos predicados son «más factivos» que otros está también presente enRosenberg (1975).

1斤 Que puede ser útil también para explicar algunas completivas de sujeto dependientes de 
En este punto no está de más precisar que el concepto de presuposición que vamos a manejar es de índole pragmática. Las presuposiciones tienen lugar en el discurso como consecuencia de la elección por parte del hablante de una determinada estrategia comunicativa. El hablante opta por tratar una porción de su mensaje como parte del saber previamente compartido por él y el oyente, es decir, como algo en cierto modo previo a esa situación comunicativa concreta, a propósito de lo cual se quiere comentar algo. Por ejemplo, en una secuencia como A mamá le preocupa que lsabel no haya llamado todavia el segmento subordinado, que Isabel no haya llamado todavía, se loma como una asunción de fondo sobre la que se comenta algo: que a mamá le preocupa ${ }^{17}$.

Preocupar es un verbo de la clase de los que vamos a denominar de afección psíquica, donde también incluiremos a encantar, extrañar, sorprender, doler, divertir, asombrar, agradar, avergonzar, alegrar, chocar, complacer, entusiasmar, molestar, apenar, afectar, etc. Desde el punto de vista del contenido tienen en común que hacen referencia a un proceso que tiene su origen en una causa y repercute en una entidad animada con capacidades mentales. El esquema sintáctico que sirve de expresión lingüística a este modo de concebir una parte de la realidad extralingüística es SUJ-PRED-CIND, donde el sujeto puede ir desempeñado por una cláusula (como en el ejemplo anterior, A mamá le preocupa que Isabel no haya llamado todavia).

La posibilidad de transmitir presuposiciones es una propiedad común a este grupo de predicados. Con verbos de afección, el hablante puede dar a entender que asume como verdadero el contenido representado en la subordinada, del cual dice que provoca un determinado estado mental (asombro, alegría, extrañe$z a$, etc.). En las secuencias de este tipo se reconocen dos partes: una que se presupone (que Isabel no haya llamado todavía) y otra que se afirma (A mamá le preocupa)

\subsection{Propiedades sintácticas.}

Las completivas en función de sujeto dependientes de predicados de afección psíquica presentan ciertas propiedades sintácticas que con frecuencia se han relacionado con la capacidad de transmitir presuposiciones. Veremos cómo, por contraste, los predicados semi-factivos (o de conocimiento) se comportan en general de manera diferente con respecto a dichas propiedades. Son las siguientes.

1) El modo de la subordinada: subjuntivo con los predicados de afección;

construcciones copulativas: Me pareció bien que se casaran. Como la problemática de las construcciones copulativas es compleja, no voy a ocuparme aquí de ellas.

17 Para que se desencadene el efecto de presuposición, es necesario que la referencia temporal del verbo subordinado sea de simultaneidad o anterioridad con respecto al predicado regente. Si la forma verbal subordinada tiene un valor temporal de posterioridad, no hay presuposición. Es sabido que el presente de subjuntivo puede expresar una referencia de presente o de futuro. 
indicativo con los de conocimiento. Ejemplos:

Me alegró / sorprendió / complació que viniera temprano [afección]

Supe / noté / me di cuenta de que venía temprano [conocimiento]

2) Posibilidad de introducir la completiva con la frase el hecho o el artículo el. El comportamiento de los verbos de conocimiento no es regular en este aspecto:

Me alegró / sorprendió / complació el que / el hecho de que viniera temprano [afección]

Ignoré / me di cuenta de *el que / el hecho de que venía temprano[conocimiento]

Supe / noté *el que / *el hecho de que venía temprano ${ }^{18}$ [conocimiento]

3) Posibilidad de anteponer la cláusula de sujeto.

Los predicados de afección llevan por lo general el sujeto pospuesto (aun en el caso de que esté desempeñado por una frase: nos sorprendió su llegada imprevista; te molestaba cualquier presencia humana, Sur 3I $)^{19}$, sin embargo, permiten su anteposición, como vemos en los siguientes ejemplos, incluso con completiva de sujeto:

\section{Que viniera de forma imprevista nos sorprendió}

Que lleve a un amigo en el pescante, ¿a quién le interesa? (His 86).

Con los verbos de conocimiento la anteposición es más forzada (aunque no imposible, vid. nota 18 ).

?(De) que venía temprano supe / noté / me di cuenta

\subsection{Completiva de sujeto y presuposición}

Las propiedades examinadas nos permiten reconocer como típicamente factivos a los predicados de afección psíquica. A continuación estudiaremos la posible relación entre estos comportamientos sintácticos y la posición estructural de sujeto. Si la función de sujeto resulta ser propicia (al menos más que otras) a la aparición de estos fenómenos, entonces podremos avanzar la hipótesis de una posible vinculación entre la capacidad de comunicar presuposiciones y el ocupar dicho hueco funcional.

18 Cita Lapesa (1984) el siguiente ejemplo de Cervantes: «Sé que [en Noruega] la mitad del año se IIeva la noche y la otra mitad el día. El que sea esto assí, yo lo sé; el por qué sea assí ignoro". Es un caso de topicalización del CDIR, donde éste pasa a ocupar la posición inicial. Además, cl modo verbal es el subjuntivo. La función de la subordinada El que sea esto assí en el discurso no es afirmar algo, sino mencionar una porción de información previamente transmitida (y que por tanto el oyente ya conoce)

1.) Téngase en cuenta que los elementos de mayor complejidad categorial (las subordinadas con verbo en forma personal) tienden a colocarse en la posición final de la cláusula (vid. Dik 1978: $\$ 9.0$ ).

20 Vid. Alarcos (1968). 
Observaremos tres funciones clausales conjuntamente: la de sujeto (SUJ), la de complemento directo (CDIR) y la de suplemento (SUPL) ${ }^{20}$. Es posible encontrar ejemplos donde un verbo de la clase de los factivos lleve una subordinada en alguna de las tres funciones:
Me alegró que viniera
(SUJ)
Lamenté que viniera
(CDIR)
Me alegré de que viniera
(SUPL)

En cualquiera de las tres funciones que ocupe la completiva, el verbo de la subordinada es el subjuntivo. Ahora bien, los resultados son más variables en lo que concierne a la introducción de la completiva por medio de el que / el hecho de que y a su posible anteposición al predicado:

Posibilidad de el que:

Me alegró el que viniera

Lamenté ?el que viniera

Me alegré *del que viniera

Anteposición:

Que viniera me alegró

?Que viniera lamenté

?De que viniera me alegrế2

(SUJ)

(CDIR)

$(\mathrm{SUPL})^{21}$

En definitiva, cuando la completiva desempeña la función de sujeto (dentro del esquema SUJ-PRED-CIND) responde positivamente a las tres propiedades sintácticas que consideramos típicas de la factividad y que se han relacionado con la presuposición: presencia del modo subjuntivo, posible anteposición y admisión de el que / el hecho de que. Otros verbos factivos que llevan cláusula subordinada en otra función sintáctica no responden uniformemente a esas tres propiedades.

Hemos avanzado que estos hechos se explican por la existencia de una vinculación entre la posibilidad de accionar presuposiciones y la posición estructural de sujeto, especialmente visible en el caso de los predicados de afección psíquica del español. Debemos ahora justificar esta relación.

El sujeto es una función sintáctica, esto es, una relación gramatical de carácter formal que se materializa por medio de ciertos procedimientos de marcación. En español estos son fundamentalmente los siguientes:

21 La presencia del artículo es considerada por Lenz (1935) y otros autores como una prueba del carácter «sustantivo» de la completiva, en especial cuando ésta desempeña la función de sujeto. Según Alarcos, «Es inusitado el artículo enfático fuera de la función de sujeto» (1963: 262).

22 Estos resultados no sorprenden si se observan a la luz de los fenómenos de orden más frecuentes en español (lengua de orden SV fundamentalmente). Pero insisto en que los predicados de afección psíquica llevan por lo general el sujeto pospuesto, y son por lo tanto VS. Desde esta perspectiva sí son significativos estos fenómenos. Vid. Pala (1974), Morales de Walters (1982), Delbecque (1987). 
- La concordancia de número entre la expresión que hace las veces de sujeto y cl predicado (que se concretiza en la desinencia verbal).

-La ausencia de marcas prepositivas (y la presencia de la forma "nominativa" de los pronombres personales).

- La posición preverbal.

Ahora bien, la conjunción de estas tres (o cuatro) marcas da lugar a los casos prototípicos, como el siguiente:

\section{Los niños abrieron los paquetes}

Pero no tienen que darse siempre juntas, y de hecho en muchas ocasiones no confluyen. Así, por ejemplo, cuando en función de sujeto hay una estrucuura coordinada, es frecuente que la forma del verbo sea singular. Por otra parte, el orden básico de constituyentes en español es SV, pero determinados tipos de predicados favorecen la posposición del sujeto (vid. nota 22).

Coinciden el mayor número de marcas de la función cuando el referente del sujeto reúne una serie de características propias de los agentes prototípicos, lo cual permite sugerir la posibilidad de una correlación entre sujetos y agentes. Particularmente, se suelen citar las siguientes ${ }^{23}$ :

La autonomía. Tiene más posibilidades de ser agente (y también de gramaticalizarse como sujeto) aquella entidad que exista con independencia del proceso en que interviene y que sea previo a él.

El ser "fuente de energía" para el proceso, esto es, estar capacitado para iniciarlo.

La volición.

El control.

De todo esto se deduce que los seres humanos son mejores candidatos a constituirse en agentes que otras entidades (particularmente, las cláusulas completivas).

Sin embargo, desde el momento en que el contenido de la subordinada es presentado por el hablante como algo previo al proceso, crecen las posibilidades de que se interprete como agente.

Por otra parte, el hecho de que ese referente proposicional se presuponga tiene que ver también con el hecho de que pueda ser concebido como el origen del proceso de afección.

Estas razones explican, a mi juicio, que los procesos de afección psíquica se gramaticalicen en español por medio del esquema SUJ-PRED-CIND, incluso cuando su causa es una entidad abstracta que se expresa por medio de una cláusula completiva, y permiten defender la tesis de que la posición de sujeto es

23. Vid. Lakoff (1977), van Oosten (1986) o Dowty (1991). 
especialmente propicia para transmitir presuposiciones ${ }^{24}$.

\section{Conclusiones}

En resumen, los predicados de afección psíquica son factivos. Funcionan como accionadores presuposicionales en la medida en que propician la interpretación del contenido de la subordinada como una presuposición compartida por el hablante y el oyente. Además, muestran propiedades sintácticas propias de los verbos típicamente factivos (esto es, los llamados por Bybee y Terrell de «comentario»): el modo de la forma verbal subordinada es el subjuntivo, admiten sin lugar a dudas la introducción de la subordinada por medio de la frase el hecho o el artículo el, y permiten el orden SV.

Por otra parte, hemos detectado una relación entre las propiedades sintácticas típicas de la factividad y el hecho de que la subordinada desempeñe la función de sujeto. De esta manera, un fenómeno sintáctico como es la utilización de una cláusula completiva en la posición gramatical de sujeto obtiene una explicación en un fenómeno de carácter discursivo (la presuposición).

\section{Referencias bibliográficas:}

Alarcos, E. (1963), Español «que», Archivum, 13; reed. en Alarcos, E. (1980), 260-274.

Alarcos, E. (1968), «Verbo transitivo, verbo intransitivo y estructura del predicado", Archivum, 18; reed. en Alarcos, E. (1980), 148-162.

Alarcos, E. (1980), Estudios de gramática funcional del español, Madrid, Gredos.

Bosque, I. (ed.) (1990), Indicativo y subjuntivo, Madrid, Taurus.

Bybee, J. y Terrell, T. (1974), "A Semantically Based Analysis of Mood in

Spanish», Hispania, 57; cito por la trad. esp. de C. Insenser, Análisis semántico del modo en español, en Bosque, I. (ed.) (1990), 145-163.

Danes, F. (ed.) (1974), Papers on Functional Sentence Perspective, La Haya / París, Mouton.

Deaño, A. (1980), Introducción a la lógica formal, Madrid, Alianza (2a ed.). Delbecque, N. (1987), Problèmes et méthodes de l'étude de la variation syntaxique.

Le cas de la position du sujet en espagnol, Lovaina, Leuven Univ. Press.

24 También es posible interpretar como presuposicionales otros sujetos en forma de cláusula completiva (dependiendo de predicados de otra índole). Por ejemplo:

"Que otra voz inesperada se sumara un momento a los susurros no tenía por qué ir más allá de un banal enigma de verano" (Gle 41).

"el que una frase dada sea gramatical o aceptable no es un hecho objetivo" (Bun 103). 
Demonte, V. (1977), La subordinación sustantiva, Madrid, Cátedra.

Dik, S.C. (1978), Functional Grammar, Amsterdam, North Holland; trad. esp. de Serrano, S. y Martín Mingorance, L., Gramática funcional, M ad rid, S.G.E.L., 1981.

Dowty, D. (1991), «Thematic proto-roles and argument selection», Language, 67/3, 547-619.

Fernández Ramírez, S. (1986), Gramática española. 4. El verbo y la oración, Madrid, Arco/Libros.

Fillmore, C.J. y Langendoen, D.T. (eds.) (1971), Studies in Linguistic Semantics, Nueva York, Holt, Rinehart and Winston, Inc..

Guitart, J.M. (1990), «Aspectos pragmáticos del modo en los complementos de predicados de conocimiento y de adquisición del conocimiento», en Bosque, I. (ed.) (1990), 315-329.

Harman, G. y Davidson, D. (eds.) (1972), Semantics of Natural Language, Dordrecht, Reidel.

Hooper, J. B. (1975), «On assertive predicates», in Kimball, J. P. (ed.), Syntax and Semantics, 4, San Diego, Academic Press, 91-124.

Karttunen, L. (1971), « Some observations on factivity», Papers in Linguistics, 4, 55-69.

Karttunen, L. (1973), «Presupposition of compound sentences», Linguistic Inquiry, 4, 169-191.

Keenan, E. L. (1971), «Two kinds of presupposition in natural language», en Fillmore, C.J. y Langendoen, D.T. (eds.) (1971), 45-52.

Kempson, R. (1975), Presupposition and the Delimitation of Semantics, Cambridge, Cambridge Univ. Press.

Kiparsky, P. y Kiparsky, C. (1971), «Fact», en Steinberg, D.D y Jakobovits, Semantics. An interdisciplinary reader in Philosophy, Linguistics and Psychology, Cambridge, Cambridge Univ. Press.

Lakoff, G. (1977), « Linguistic Gestalts», Papers from the Thirteenth Regional Meeting, Chicago, CLS, 236-287.

Lapesa, R. (1984), «El uso de actualizadores con el infinitivo y la suboración sustantiva en español: diacronía y sentido», en Schwartz Lerner, L. y Lerner, I. (eds.) (1984), 65-89.

Lenz, R. (1935), La oración y sus partes, Madrid, Centro de estudios históricos. Levinson, S. C. (1983), Pragmatics, Cambridge, Cambridge Univ. Press; cito por la trad. esp. de A. Rubiés, Pragmática, Barcelona, Teide, 1989.

Martínez, J. A. (1984), «Construcciones ecuacionales: un dilema de gramática normativa», Actas del II Simposio Internacional de Lengua Española (1981), Madrid, Cabildo Insular de Gran Canaria, 99-112.

Morales de Walters, A. (1982), «La posición de sujeto en el español de Pucrto Rico a la luz de la clase semántica verbal, la oposición tema-rema y el tópico oracional», L.E.A., 4, 23-38. 
Oh, C. K. (1974), «More on Degrees of Factivity», Papers from the Tenth Regional Meeting, Chicago, CLS, 517-528.

Pala, K. (1974), «Semantic classes of verbs and FSP», en Danes, F. (ed.) (1974), 196-207.

Rosenberg, M.S. (1975), Counterfactives: a pragmatic analysis of presupposition, Ann Arbor, UMI.

Schwartz Lerner, L. y Lerner, I. (eds.) (1984), Homenaje a Ana Ma Barrenechea, Madrid, Castalia.

Stalnaker, R.C. (1972), «Pragmatics», en Harman, G. y Davidson, D. (eds.) (1972), 380-397.

Strawson, P. F. (1950), «On referring», Mind, 59: trad. esp. de Luis MI.

Valdés, «Sobre el referir», en Luis Ml. Valdés (ed.), La búsqueda del significado, Madrid, Tecno / Univ. de Murcia, 1991, 57-82.

van Oosten, J. (1986), The Nature of Subjects, Topics and Agents: a Cognitive Explanation, Indiana, Indiana University Linguistics Club.

Abreviaturas empleadas en los ejemplos:

Bun = Bunge, M., Lingüística y filosofía, Barcelona, Ariel, 1983.

Gle = Cortázar, J., Queremos tanto a Glenda, Madrid, Alfaguara, 1981 ( $4^{\mathbf{a}}$ ed.).

His = Bioy Casares, A., Historias desaforadas, Madrid, Alianza, 1986.

Sur = García Morales, A., El Sur (seguido de Bene), Barcelona, Anagrama, 1985. 